EPJ Web of Conferences 60, 04004 (2013)

DOI: $10.1051 /$ epjconf/20136004004

(C) Owned by the authors, published by EDP Sciences, 2013

\title{
Charm physics
}

\author{
J. van Tilburg ${ }^{1, a}$ on behalf of the LHCb Collaboration \\ ${ }^{1}$ Physikalisches Institut Heidelberg, Germany
}

\begin{abstract}
Charm physics is an active and productive field at hadron colliders. In these proceedings an overview of recent results in charm physics from the Tevatron and LHC experiments is given. Highlighted are the measurements of open charm production and production asymmetries, the recent precision measurements of the $D$ masses, the confirmation of mixing in the $D^{0}$ system, the searches for $C P$ violation in the charm system and the searches for rare charm decays.
\end{abstract}

\section{Introduction}

The ambition in heavy flavour physics is to find anomalous effects in loop diagrams that may reveal the presence from yet unknown, heavy particles. Measurements of $C P$ violation and searches for rare decays are particularly sensitive to these effects. Such indirect searches for new particles are complementary to the direct searches at general purpose detectors.

Although most experimental effort is focussed on measuring the properties of beauty hadrons, new physics can equally well reveal itself in the charm sector. Moreover, charm decays are sensitive to other flavour couplings compared to $B$ decays. The mass of the charm quark is large enough to be considered as heavy in theoretical calculations, opening up the possibility for (relatively) precise predictions of the Standard Model processes. Since the charm cross section is about a factor twenty above the beauty cross section, huge amounts of charm decays are now available at the hadron colliders. This allows to make high-precision tests of the Standard Model.

Charm physics is a broad field and for a long time it has been dominated by electron collider experiments. With the availability of large samples of charm decays, hadron collider experiments come more into play in this field. These proceedings focus on recent results from the LHC and Tevatron, in particular open charm production and production asymmetries, $D$ mass measurements, $D^{0}$ mixing, $C P$ violation in the charm system and rare charm decays.

\section{Open charm production and production asymmetries}

The production of open charm in hadronic collisions at new energy scales provides an important measure for our understanding of quantum chromodynamics (QCD). In addition, a good knowledge of charm production is needed

\footnotetext{
a e-mail: jeroen.van.tilburg@ cern.ch
}

for background estimates of Standard Model processes, such as Higgs production. Production measurements are a powerful test of QCD calculation at next-to-leading order (NLO). The QCD@NLO calculations can be done in the framework of Fixed-Order Next-to-Leading Logarithm [1] (FONLL) or in the framework of Generalized Mass Variable Flavour Number Scheme [2] (GM-VFNS).

Several new production measurements at LHC energies have been performed over the past years: at $\mathrm{LHCb}$ at $7 \mathrm{TeV}$ using exclusive final states [3] and using inclusive final states in the high- $p_{\mathrm{T}}$ region [4], at ALICE using exclusive final states at 2.76 and $7 \mathrm{TeV}$ [5] and inclusive (electron) states at $7 \mathrm{TeV}$ [6], and at ATLAS at $7 \mathrm{TeV}$ using exclusive final states [7] and in jets using $D^{*}$ final states [8]. The measured cross sections are typically above the FONLL and below the GM-VFNS predictions, but in general they are in good agreement with the QCD calculations. An overview of open charm cross sections in hadronic collisions at several centre-of-mass energies is shown in Fig. 1, where the measurements are compared to NLO calculations from Ref. [9].

For precise measurements of $C P$ violation a good understanding of production asymmetries is needed. The production asymmetry is defined as the difference in production cross section between $D$ mesons and $\bar{D}$ mesons over their sum,

$$
A_{P}=\frac{\sigma(D)-\sigma(\bar{D})}{\sigma(D)+\sigma(\bar{D})}
$$

In many analyses that search for $C P$ violation these production asymmetries need to be subtracted from the observed charge asymmetry. Due to the initial state, these production asymmetries are more relevant at $p p$ colliders than at $p \bar{p}$ colliders. Even though the production mechanism is charge symmetric - charm quarks are always produced in $c \bar{c}$ pairs in the strong interaction - the hadronisation process can cause a small production asymmetry. The main effect is coming from beam drag, which is caused 


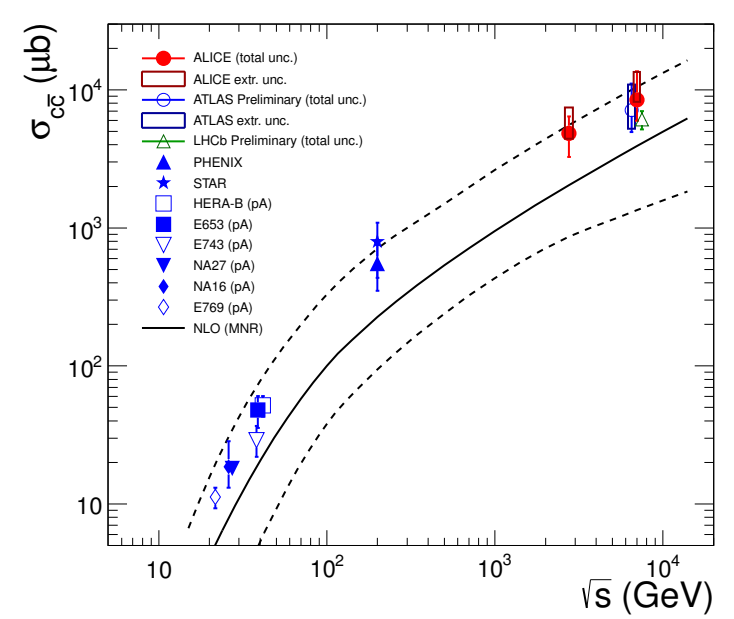

Figure 1. Open charm cross sections in hadronic collisions versus the centre-of-mass energy. The proton-ion and deuteriumion collisions are scaled down to the number of binary collisions using the Glauber model. Figure taken from Ref. [5].

by a colour connection between the $c$ quark and the beam remnants. The $c$ quark prefers to form a baryon with the beam remnants, while the $\bar{c}$ quark prefers form a meson, giving $\sigma(\bar{D})>\sigma(D)$ and thus $A_{P}<0$. Other hadronisation effects, which could depend on $p_{T}$ or rapidity, can also play a role in this mechanism.

The $D_{s}^{ \pm}$production asymmetry has been measured [10] at $\mathrm{LHCb}$ using the $D_{s}^{ \pm} \rightarrow \phi\left(K^{+} K^{-}\right) \pi^{ \pm}$mode. Since this is a Cabibbo-favoured mode, the intrinsic $C P$ violation is expected to be negligible. On the other hand, the asymmetry coming from the detection and reconstruction of the charged pion in the final state need to be measured independently. This is done by measuring the efficiency ratio of $\pi^{+}$over $\pi^{-}$in partially reconstructed $D^{* \pm}$-tagged $D^{0} \rightarrow K^{-} \pi^{+} \pi^{+} \pi^{-}$decays where one of the three pions is missing. No large asymmetry is found in this method and the efficiency ratio is compatible with one. The production asymmetry in the rapidity region $2<y<4.5$ and for $p_{T}>2 \mathrm{GeV}$ is measured with sub-percent precision to be $A_{P}=(-0.33 \pm 0.22 \pm 0.10) \%$. In a similar fashion the $D^{ \pm}$production asymmetry has been measured more recently [11] in the Cabibbo-favoured $D^{+} \rightarrow$ $K_{S}^{0}\left(\pi^{+} \pi^{-}\right) \pi^{+}$mode. The production asymmetry in the region $2<y<4.75$ and $2<p_{T}<18 \mathrm{GeV}$ is measured to be $A_{P}=(-0.96 \pm 0.26 \pm 0.18) \%$, where a small correction coming from the neutral kaon asymmetry is applied. The negative sign in both measurements indicates the presence of beam drag effects.

\section{D mass measurements}

In contrast to $B$ mesons, relatively few precision measurements of $D$ meson masses exist. In particular the knowledge on the $D^{+}$and $D_{s}^{+}$mass is relatively limited. The uncertainty on the $D_{s}^{+}$mass currently limits the precision on the $B_{c}^{+}$mass in the $B_{c}^{+} \rightarrow J / \psi D_{s}^{+}$channel. Precise knowl- edge on the $D^{0}$ mass can also shed light on the nature of $X(3872)$ particle: whether or not it is $D^{0} D^{* 0}$ molecule.

Recent measurements [12] of the $D^{0}, D^{+}$and $D_{s}^{+}$meson masses have been performed by LHCb. Using the low Q-value modes $D^{0} \rightarrow K^{-} K^{+} K^{-} \pi^{+}, D^{0} \rightarrow K^{-} K^{+} \pi^{-} \pi^{+}$and $D_{(s)}^{+} \rightarrow K^{+} K^{-} \pi^{+}$, the main systematic uncertainty which is coming from the momentum scale and the energy loss correction is reduced. The momentum scale is calibrated using $B^{+} \rightarrow J / \psi K^{+}$and $B^{+} \rightarrow J / \psi K^{+} \pi^{-} \pi^{+}$decays. The results are summarised in Table 1 . It can be seen that the precision on the $D^{0}$ mass is similar to the best previous measurement ${ }^{1}$ from the CLEO collaboration. The result on the $D^{+}$mass is the first reported result in more than 30 years and is a factor three better than the PDG average. Finally, the precision on the $D_{s}^{+}$mass constitutes a factor five improvement on the PDG average and will reduce the uncertainty on the $B_{c}^{+}$mass.

\section{$4 D^{0}$ mixing}

In neutral meson systems mixing can occur between the particle and anti-particle state. Mixing is well-established and plays a significant role in the $K^{0}$, the $B^{0}$ and $B_{s}^{0}$ systems. On the other hand, in the $D^{0}$ system mixing is expected to be small and dominated by long-range contributions, which makes a precise prediction of the size of the mixing hard. By combining several measurements [18] $D^{0}$ mixing could be established, even though there was no single experiment with a significance of more than five standard deviations.

Recently, the LHCb and CDF collaborations both have reported clear observations $[19,20]$ of $D^{0}$ mixing which exclude the no-mixing hypothesis at $9.1 \sigma$ and $6.1 \sigma$, respectively. They have measured the time-dependent ratio of wrong-sign to right-sign $D^{0} \rightarrow K^{-} \pi^{+}$decays. Wrong-sign decays (i.e., $D^{0} \rightarrow K^{+} \pi^{-}$) can occur either through $D^{0}$ mixing followed by Cabibbo-favoured (CF) decay, through doubly-Cabibbo-suppressed (DCS) decay or through the interference between these two effects. The DCS rate $\left(R_{D}\right)$ is constant as function of the decay time, but the mixing causes the wrong-sign to right-sign ratio to have a time dependence. The mixing parameters, $x^{\prime}$ and $y^{\prime}$ are rotated by the strong phase between DCS and CF amplitudes compared to the usual mixing parameters, $x$ and $y$. The LHCb central values are $R_{D}=(3.52 \pm 0.15) \times 10^{-3}$, $y^{\prime}=(7.2 \pm 2.4) \times 10^{-3}$ and $x^{\prime 2}=(-0.09 \pm 0.13) \times 10^{-3}$, and the (preliminary) CDF ones are $R_{D}=(3.51 \pm 0.35) \times 10^{-3}$, $y^{\prime}=(4.3 \pm 4.3) \times 10^{-3}$ and $x^{\prime 2}=(0.08 \pm 0.18) \times 10^{-3}$. Due to the large correlation between $y^{\prime}$ and $x^{\prime 2}$ the $D^{0}$ mixing does not appear as significant in these central values. This correlation can be clearly seen in Fig. 2 which shows the $68 \%$ confidence contours from the $\mathrm{LHCb}$ and $\mathrm{CDF}$ results as well as those from BaBar [21] and Belle [22].

\section{$5 C P$ violation in charm}

Even though $C P$ violation is now well established in the kaon and $B$ decays, no $C P$ violation has yet been observed

\footnotetext{
${ }^{1}$ In the mean time preliminary updates on the $D^{0}$ masses are available [17].
} 
Table 1. LHCb measurements, compared to the best previous measurements and to the results of a global fit to the available open charm mass data. All values are in $\mathrm{MeV} / \mathrm{c}^{2}$.

\begin{tabular}{crrr}
\hline Quantity & \multicolumn{1}{c}{$\begin{array}{c}\text { LHCb } \\
\text { measurement }\end{array}$} & \multicolumn{1}{c}{$\begin{array}{c}\text { Best previous } \\
\text { measurement }\end{array}$} & \multicolumn{1}{c}{ PDG fit [13] } \\
\hline$M\left(D^{0}\right)$ & $1864.75 \pm 0.19$ & $1864.85 \pm 0.18[14]$ & $1864.86 \pm 0.13$ \\
$M\left(D^{+}\right)-M\left(D^{0}\right)$ & $4.76 \pm 0.14$ & $4.7 \pm 0.3[15]$ & $4.76 \pm 0.10$ \\
$M\left(D_{s}^{+}\right)-M\left(D^{+}\right)$ & $98.68 \pm 0.05$ & $98.4 \pm 0.3[16]$ & $98.88 \pm 0.25$ \\
\hline
\end{tabular}

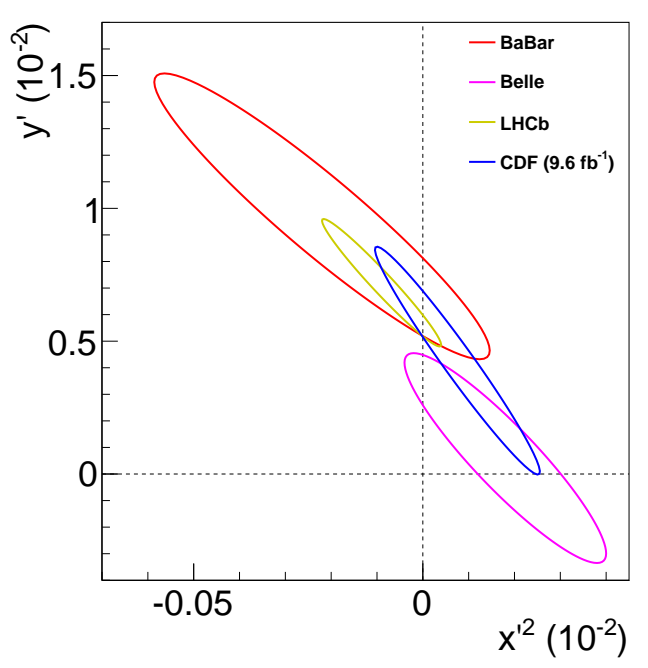

Figure 2. Comparison of the $D^{0}$ mixing $x^{\prime 2}-y^{\prime}$ contours at $68 \%$ confidence level between different experiments. Figure taken from Ref. [20].

in charm system. It is interesting to search for $C P$ violation in charm decays, since the flavour-changing-neutralcurrent processes here proceed with up-type quarks instead of down-type quarks as in the case of kaon and $B$ decays. In singly-Cabibbo-suppressed decays, direct $C P$ violation is possible through the interference between tree and penguin diagrams. Naively, this direct $C P$ violation is expectated to be at the $0.1 \%$ level $[23,24]$ in the Standard Model, while indirect $C P$ violation is predicted to be much smaller.

By measuring the difference in the $C P$ asymmetries $\left(\triangle A_{C P}\right.$ ) between $D^{0} \rightarrow K^{-} K^{+}$and $D^{0} \rightarrow \pi^{-} \pi^{+}$decays the detection and production asymmetry cancel at first order. In addition, in the Standard Model the indirect $C P$ violation is expected to be universal for all $D^{0}$ decay modes, while the direct $C P$ violation is expected to have opposite sign under $\mathrm{SU}(3)$ flavour symmetry. This makes $\Delta A_{C P}$ mainly a measurement of direct $C P$ violation.

After the measurements from the BaBar, LHCb, CDF and Belle collaborations [25-28], the world average [18], neglecting indirect $C P$ violation, yielded $\Delta A_{C P}=(-0.68 \pm$ $0.15) \%$, being 4.6 standard deviations away from the hypothesis of no $C P$ violation and larger than naive expectations. This sparked a theoretical debate on whether or not

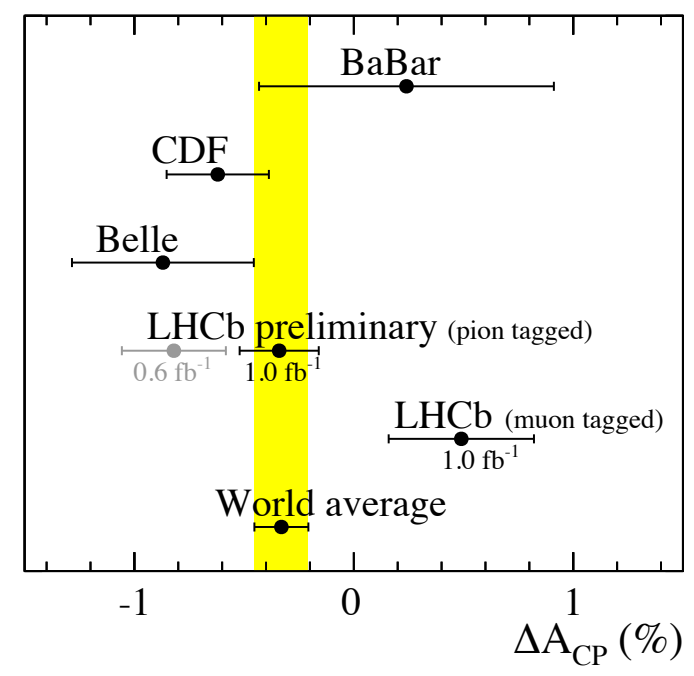

Figure 3. Comparison of different measurements of $\Delta A_{C P}$, neglecting possible effects from $C P$ violation in $D^{0}$ mixing.

this could be accommodated within the Standard Model. For a comprehensive review see Ref. [29].

Recently, LHCb has performed two independent measurements of $\triangle A_{C P}$. The first is a preliminary update of the previous result [30]. This analysis uses $D^{*+} \rightarrow D^{0} \pi^{+}$ decays to tag the flavour of the $D^{0}$. The result gives $\Delta A_{C P}=(-0.34 \pm 0.15 \pm 0.10) \%$, where the first error denotes the statistical uncertainty and the second the systematic uncertainty. The second measurement [31] of $\Delta A_{C P}$ is a new measurement, which uses the charge of the muon in semileptonic $B$ decays to tag the flavour of the $D^{0}$. This gives $\Delta A_{C P}=(+0.49 \pm 0.30 \pm 0.14) \%$. Both results do not (yet) confirm $C P$ violation in the charm sector. When including them in the world average [18] the value becomes $\Delta A_{C P}=(-0.33 \pm 0.12) \%$, which is closer to zero, but still almost three standard deviations away from the hypothesis of no $C P$ violation. A comparison of the different measurements of $\triangle A_{C P}$ is shown in Fig. 3.

Similarly, direct $C P$ violation is also possible in charged $D$ mesons. The LHCb collaboration has measured direct $C P$ violation in the singly-Cabibbo-suppressed $D^{+} \rightarrow \phi \pi^{+}$and $D_{s}^{+} \rightarrow K_{S}^{0} \pi^{+}$decay modes [32]. This analysis uses the Cabibbo-favoured modes $D^{+} \rightarrow K_{S}^{0} \pi^{+}$and $D_{s}^{+} \rightarrow \phi \pi^{+}$to subtract the production and detection asymmetry. Additionally, the variation in $C P$ violation across the $\phi$ mass in the $D^{+} \rightarrow K^{+} K^{-} \pi^{+}$Dalitz plane can be measured, which is denoted as $A_{C P} \mid S$. The $C P$ asymmetries 


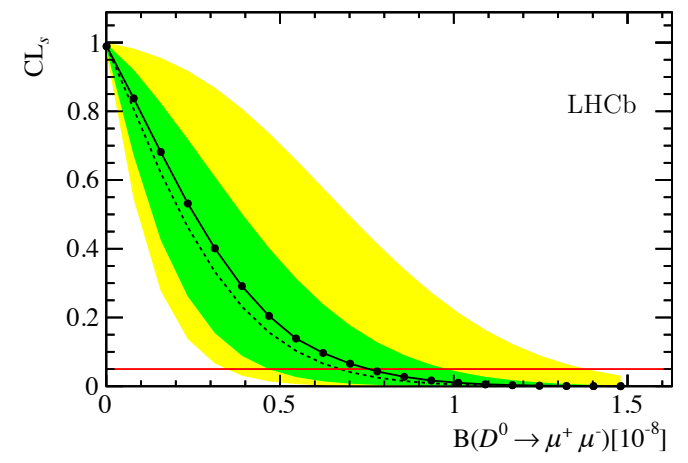

Figure 4. $\mathrm{CL}_{S}$ exclusion plot (solid line) as a function of the assumed $D^{0} \rightarrow \mu^{+} \mu^{-}$branching fraction and median (dashed line), $1 \sigma$ and $2 \sigma$ bands of the expected $\mathrm{CL}_{S}$, in the background-only hypothesis, obtained with the asymptotic $\mathrm{CL}_{S}$ method. The horizontal line corresponding to $\mathrm{CL}_{S}=0.05$ is also drawn. Figure taken from Ref. [34].

are measured to be

$$
\begin{aligned}
A_{C P}\left(D^{+} \rightarrow \phi \pi^{+}\right) & =(-0.04 \pm 0.14 \pm 0.13) \%, \\
A_{C P} \mid S\left(D^{+} \rightarrow \phi \pi^{+}\right) & =(-0.18 \pm 0.17 \pm 0.18) \%, \\
A_{C P}\left(D_{s}^{+} \rightarrow K_{S}^{0} \pi^{+}\right) & =(+0.61 \pm 0.83 \pm 0.13) \%,
\end{aligned}
$$

where the first error denotes the statistical uncertainty and the second the systematic uncertainty. No $C P$ violation is observed in these modes.

\section{Rare charm decays}

Searching for rare decays of charm hadrons has similar sensitivity for the presence of new particles in loop diagrams as in the case of $B$ hadrons. One of the key channels is the rare $D^{0} \rightarrow \mu^{+} \mu^{-}$decay. The Standard Model predicts the branching ratio to be below $6 \times 10^{-11}$. This limit is dominated by long-distance contributions, in particular the two-photon intermediate state. The best previous limit was set by the Belle collaboration, which measured $\mathcal{B}\left(D^{0} \rightarrow \mu^{+} \mu^{-}\right)<1.4 \times 10^{-7}$ at $90 \%$ confidence level [33].

This result has recently been overtaken by the $\mathrm{LHCb}$ collaboration. The analysis [34] searches for $D^{0} \rightarrow \mu^{+} \mu^{-}$ decays coming from $D^{*+} \rightarrow D^{0} \pi^{+}$decays. As normalization and calibration channels $J / \psi \rightarrow \mu^{+} \mu^{-}, D^{0} \rightarrow \pi^{+} \pi^{-}$ and $D^{0} \rightarrow K^{-} \pi^{+}$decays are used. The branching ratio is found to be below $7.6 \times 10^{-9}$ at $95 \%$ confidence level, which is more than a factor 20 improvement with respect to the Belle result. Figure 4 shows the $\mathrm{CL}_{S}$ exclusion plot.

A family of rare decays that have very similar topologies is denoted by $D_{(s)}^{+} \rightarrow \pi \mu \mu$. The first decay, $D^{+} \rightarrow$ $\pi^{+} \mu^{+} \mu^{-}$, is a flavour-changing-neutral-current (FCNC) decay, which has a predicted banching ratio of about $1.3 \times$ $10^{-9}$. The second decay, $D_{s}^{+} \rightarrow \pi^{+} \mu^{+} \mu^{-}$, can occur through weak annihilition. This channel can be used to normalise a potential signal in the $D^{+}$mode where an analogous weak annihilation diagram exist, which is suppressed by a factor $\left|V_{c d}\right|^{2}$. Finally, the third decay, $D_{(s)}^{+} \rightarrow$ $\pi^{-} \mu^{+} \mu^{+}$, is a lepton-number-violating decay and is forbidden by the Standard Model. The LHCb collaboration has measured [35] at 95\% confidence level and excluding resonant regions

$$
\begin{aligned}
& \mathcal{B}\left(D^{ \pm} \rightarrow \pi^{ \pm} \mu^{+} \mu^{-}\right)<8.3 \times 10^{-8}, \\
& \mathcal{B}\left(D_{s}^{ \pm} \rightarrow \pi^{ \pm} \mu^{+} \mu^{-}\right)<4.8 \times 10^{-7}, \\
& \mathcal{B}\left(D^{ \pm} \rightarrow \pi^{\mp} \mu^{ \pm} \mu^{ \pm}\right)<2.5 \times 10^{-8}, \\
& \mathcal{B}\left(D_{s}^{ \pm} \rightarrow \pi^{\mp} \mu^{ \pm} \mu^{ \pm}\right)<1.4 \times 10^{-7},
\end{aligned}
$$

improving the best previous limits by several orders of magnitude.

\section{Conclusion and outlook}

With many more analyses on-going, charm physics is an active field at hadron colliders. In particular, more results on direct and indirect $C P$ violation in charm decays can be expected in the near future. The strength of hadron colliders is estabilished by the huge statistical samples of charm decays, giving high sensitivity to small $C P$ violation effects and access to very rare decays. Charm physics at hadron colliders is delivering already many results. The $\mathrm{LHCb}$ measurements given in these proceedings have used only the 2011 data set and about three times more data is currently available for analysis. Therefore, more interesting results on charm physics are expected in the near future.

\section{References}

[1] M. Cacciari et al., J. High Energy Phys. 1210, 137 (2012).

[2] B. Kniehl et al., Eur. Phys. J. C 72, 2082 (2012).

[3] LHCb collaboration, R. Aaij et al., Nucl.Phys. B 871, 1 (2013) and LHCb collaboration, "Prompt charm production in $p p$ collisions at $\sqrt{s}=7 \mathrm{TeV}$," LHCbCONF-2010-013.

[4] LHCb collaboration, "Measurement of $\sigma(b \bar{b})$ with inclusive final states," LHCb-CONF-2013-002.

[5] ALICE collaboration, B. Abelev et al., J. High Energy Phys. 1207, 191 (2012).

[6] ALICE collaboration, B. Abelev et al., Phys. Lett. B 721, 13 (2013).

[7] ATLAS collaboration, "Measurement of $D^{*}$ meson production cross sections in pp collisions at $\sqrt{s}=$ $7 \mathrm{TeV}$ with the ATLAS detector," ATLAS-CONF2011-017.

[8] ATLAS collaboration, G. Aad et al., Phys. Rev. D 85, 052005 (2012).

[9] M. L. Mangano, P. Nason and G. Ridolfi, Nucl. Phys. B 373, 295 (1992).

[10] LHCb collaboration, R. Aaij et al., Phys. Lett. B 713, 186 (2012).

[11] LHCb collaboration, R. Aaij et al., Phys. Lett. B 718, 902 (2013). 
[12] LHCb collaboration, R. Aaij et al., J. High Energy Phys. 06, 065 (2013).

[13] Particle Data Group, J. Beringer et al., Phys. Rev. D 86, 010001 (2012).

[14] CLEO collaboration, C. Cawlfield et al., Phys. Rev. Lett. 98, 092002 (2007).

[15] R. Schindler et al., Phys. Rev. D 24, 78 (1981).

[16] BaBar collaboration, B. Aubert et al., Phys. Rev. D 65, 091104 (2002).

[17] A. Tomaradze et al., "Is the Exotic Hadron $X(3872)$ a $D^{0} D^{* 0}$ Molecule: Precision Determination of the Binding Energy of $X(3872)$," arXiv:1212.4191.

[18] Y. Amhis et al., "Averages of b-hadron, chadron, and tau-lepton properties as of early 2012," arXiv:1207.1158 and online update at http://www.slac.stanford.edu/xorg/hfag.

[19] LHCb collaboration, R. Aaij et al., Phys. Rev. Lett. 110, 101802 (2013).

[20] CDF collaboration, "Observation of $D^{0}-\overline{D^{0}}$ Mixing Using the CDF II Detector", CDF Public Note 10990.

[21] BaBar collaboration, B. Aubert et al., Phys. Rev. Lett. 98, 211802 (2007).

[22] Belle collaboration, M. Staric et al., Phys. Rev. Lett. 98, 211803 (2007).

[23] S. Bianco, F. Fabbri, D. Benson, and I. Bigi, Riv. Nuovo Cim. 26N7, 1 (2003).
[24] Y. Grossman, A. L. Kagan, and Y. Nir, Phys. Rev. D 75, 036008 (2007).

[25] BaBar collaboration, B. Aubert, et al., Phys. Rev. Lett. 100, 061803 (2008).

[26] LHCb collaboration, R. Aaij, et al., Phys. Rev. Lett. 108, 111602 (2012).

[27] CDF collaboration, T. Aaltonen, et al., Phys. Rev. Lett. 109, 111801 (2012).

[28] B. Ko, "Direct CP violation in charm at Belle," arXiv:1212.1975.

[29] LHCb collaboration, R. Aaij, et al., Eur. Phys. J. C 73, 2373 (2013).

[30] LHCb collaboration, "A search for time-integrated $C P$ violation in $D^{0} \rightarrow K^{-} K^{+}$and $D^{0} \rightarrow \pi^{-} \pi^{+}$decays", LHCb-CONF-2013-003.

[31] LHCb collaboration, R. Aaij, et al., Phys. Lett. B 723, 33 (2013).

[32] LHCb collaboration, R. Aaij et al., "Search for CP violation in $D^{+} \rightarrow \phi \pi^{+}$and $D_{s}^{+} \rightarrow K_{S}^{0} \pi^{+}$decays", to appear in J. High Energy Phys., arXiv:1303.4906.

[33] Belle collaboration, M. Petric et al., Phys. Rev. D 81, 091102 (2010).

[34] LHCb collaboration, R. Aaij et al., "Search for the rare decay $D^{0} \rightarrow \mu^{+} \mu^{-}$, , to appear in Phys. Lett. B, arXiv:1305.5059.

[35] LHCb collaboration, R. Aaij et al., "Search for $D_{(s)}^{+} \rightarrow \pi^{+} \mu^{+} \mu^{-}$and $D_{(s)}^{+} \rightarrow \pi^{-} \mu^{+} \mu^{+}$decays," to appear in Phys. Lett. B, arXiv:1304.6365. 